\title{
DIRICHLET-FINITE OUTER FUNCTIONS
}

\author{
SHINJI YAMASHITA
}

(Received 28 April 1980)

Communicated by E. Strzelecki

\begin{abstract}
Given a measurable function $k$ non-negative a.e. on the circle $|z|=1$, when is the outer function $T_{k}$ (see (1.3)) continuous on the disk $|z|<1$ and further, Dirichlet-finite: $\iint_{|z|<1 \mid}\left|T_{k}^{\prime}(z)\right|^{2} d x d y<\infty$ ? We shall show, among other results, that the answer is in the positive if $k \in \Lambda_{\alpha}^{2}, \frac{1}{2}<\alpha<1$, with ess inf $k>0$.

1980 Mathematics subject classification (Amer. Math. Soc.): primary 30 E 20, 30 E 25; secondary 31 A 10,31 A 20,31 A 25 .
\end{abstract}

\section{Introduction}

By the Dirichlet integral $D(f)$ of a function $f$ analytic in $U=\{|z|<1\}$ we mean

$$
D(f)=\iint_{U}\left|f^{\prime}(z)\right|^{2} d x d y \quad(z=x+i y) .
$$

Let $\mathscr{D}$ be the family of Dirichlet-finite analytic functions $f$ in $U$, namely, $D(f)<\infty$. Then $f \in \mathscr{D}$ has the finite angular limit $f^{*}\left(e^{i t}\right)$ at a.e. (almost every(where)) point $e^{i t}$ of the unit circle $C=\{|z|=1\}$, and that $f^{*}$ is a member of complex $L^{p}(C)$ for all $p, 0<p<\infty$. J. A. Cima [3] raised the following question:

Given a $k \in \cap_{p>0} L^{p}(C)$, non-negative a.e., when can we find an $f \in \mathscr{D}$ such that

$$
\left|f^{*}\left(e^{i t}\right)\right|=k\left(e^{i t}\right) \text { a.e. on } C \text { ? }
$$

OCopyright Australian Mathematical Society 1981 
The above problem is a part of the following:

(Q1) Find a necessary and sufficient condition for $k \geqslant 0$, a.e. on $C$, so that there exists $f \in \mathscr{D}$ satisfying (1.1).

This is essentially answered by L. Carleson [1].

TheOrem 1 (Carleson [1]). A necessary and sufficient condition on $k$ for (Q1) is that, $k$ is measurable, that $\log k \in L^{1}(C)$, and that

$$
J(k) \equiv \int_{0}^{\pi} \frac{d t}{\sin ^{2} \frac{t}{2}} \int_{-\pi}^{\pi}\left[\log \frac{k\left(e^{i(s+t)}\right)}{k\left(e^{i s}\right)}\right]\left[k\left(e^{i(s+t)}\right)^{2}-k\left(e^{i s}\right)^{2}\right] d s<\infty
$$

In effect, the necessity of (1.2) follows from Carleson's result [1, Theorem]. To prove the sufficiency we let $\operatorname{Exp} L^{1}$ be the family of measurable functions $k>0$ a.e. on $C$ such that $\log k \in L^{1}(C)$. By an outer function we mean an analytic function $T_{k}$ in $U$ defined by

$$
T_{k}(z)=\exp \left[\frac{1}{2 \pi} \int_{-\pi}^{\pi} \frac{e^{i t}+z}{e^{i t}-z} \log k\left(e^{i t}\right) d t\right], \quad z \in U,
$$

where $k \in \operatorname{Exp} L^{1}$. Then the angular limit $T_{k}^{*}\left(e^{i t}\right)$ exists and

$$
\left|T_{k}^{*}\left(e^{i t}\right)\right|=k\left(e^{i t}\right) \quad \text { a.e. on } C \text {. }
$$

Returning to Theorem 1 we assume (1.2). It follows from the inequality [1, (7)] (this is used, in effect, for the proof of his main theorem by Carleson, yet [1, Section 4] is self-contained) that $D\left(T_{k}\right)<(8 \pi)^{-1} J(k)<\infty$.

Since the outer function is considered in the proof of the sufficiency of (1.2), it is natural to restrict the family $\mathscr{D}$ within outer functions. More restrictively, let D $*$ be the family of $T_{k} \in \mathscr{D}$ such that $T_{k}$ can be extended continuously to $\bar{U}=U \cup C$, or, continuous on $\bar{U}$, for short.

(Q2) For what function $k \geqslant 0$ a.e. on $C$, is $T_{k} \in \mathscr{D} *$ true?

We begin with continuous $k$. Let $\Lambda_{\alpha}(0<\alpha \leqslant 1)$ be the family of complexvalued functions $k$ on $C$ such that

$$
\left|k\left(e^{i t}\right)-k\left(e^{i s}\right)\right|<A_{k}\left|e^{i t}-e^{i s}\right|^{\alpha} \quad \text { on } C,
$$

where $A_{k} \geqslant 0$ is a constant [4, p. 72]; apparently, $\left|e^{i t}-e^{i s}\right|^{\alpha}$ in the definition may be replaced by $|t-s|^{\alpha}, t, s \in(-\infty,+\infty)$. 
THEOREM 2. Let $k \in \Lambda_{\alpha}(0<\alpha<1)$ be positive on $C$. Then $T_{k}$ is continuous on $\bar{U}$ such that

$$
\left|T_{k}^{*}\left(e^{i t}\right)\right|=k\left(e^{i t}\right) \text { for all } e^{i t} \in C
$$

Furthermore,

$$
T_{k}^{\prime}(z)=O\left((1-|z|)^{-1+\alpha}\right) \text { as }|z| \rightarrow 1-0 .
$$

In the special case $\frac{1}{2}<\alpha<1$, it follows from (1.6) that $T_{k} \in \mathscr{D}^{*}$. K.-N. Chow and D. Protas [2, Theorem 2.2] proved that if $k \in \Lambda_{\beta}\left(\frac{1}{2}<\beta<1\right)$ is positive, then $D\left(T_{k}\right)<\infty$ and (1.4) holds. We can drop the word "almost" in (1.4) in the preceding sentence. Actually, choose $\frac{1}{2}<\alpha<\beta$. Then $k \in \Lambda_{\alpha}$ because $\Lambda_{\beta} \subset$ $\Lambda_{\alpha}$. Therefore, (1.5) and $D\left(T_{k}\right)<\infty$ are true.

Let $\Lambda_{\alpha}^{p}(1<p<\infty, 0<\alpha \leqslant 1)$ be the family of complex $k \in L^{p}(C)$ such that

$$
\sup _{0<h<t} \int_{-\pi}^{\pi}\left|k\left(e^{i(s+h)}\right)-k\left(e^{i s}\right)\right|^{p} d s=O\left(t^{p \alpha}\right) \quad \text { as } t \rightarrow 0
$$

see [4, p. 72]. Then $\Lambda_{\alpha} \subset \Lambda_{\alpha}^{p}$ for $1<p<\infty, 0<\alpha<1$.

THEOREM 3. Let $1<p<\infty$ and $1 / p<\alpha<1$. Suppose that $k \in \Lambda_{\alpha}^{p}$ is real and there exists a constant $m>0$ such that $k>m$ a.e. on $C$. Then $T_{k}$ is continuous on $\bar{U}$ with (1.4). Furthermore,

$$
\int_{-\pi}^{\pi}\left|T_{k}^{\prime}\left(r e^{i t}\right)\right|^{p} d t=O\left((1-r)^{-p+p \alpha}\right) \text { as } r \rightarrow 1-0 .
$$

Especially if $p=2$ and $\frac{1}{2}<\alpha<1$, then $T_{k} \in \mathscr{D}^{*}$ by (1.7). Therefore, the best answer to (Q2) in the present paper is that $k \in \Lambda_{\alpha}^{2}\left(\frac{1}{2}<\alpha<1\right)$ with ess inf $k>0$.

\section{Proofs}

For the proof of Theorem 2, let

$$
0<m \leqslant k\left(e^{i t}\right)<M<\infty \text { for all } e^{i t} \in C .
$$

Then, $T_{k}=M \exp g$, where

$$
g(z)=\frac{1}{2 \pi} \int_{-\pi}^{\pi} \frac{e^{i t}+z}{e^{i t}-z} \log \frac{k\left(e^{i t}\right)}{M} d t .
$$

Since $1 / k \leqslant 1 / m$, it follows that $\log (k / M) \in \Lambda_{\alpha}$. It further follows from [4, Theorem 5.8, p. 83] that $g$ is continuous on $\bar{U}$ with $g^{*} \in \Lambda_{\alpha}$. Therefore $T_{k}$ is continuous on $\bar{U}$ with $\left|T_{k}^{*}\right|=M \exp \left(\operatorname{Re} g^{*}\right)=k$ everywhere on $C$. To observe 
(1.6) we note that

$$
g^{\prime}(z)=O\left((1-|z|)^{-1+\alpha}\right)
$$

by $\left[4\right.$, Theorem 5.1, p. 74]. Since $\operatorname{Re} g \leq 0$, it follows that $\left|T_{k}^{\prime}\right|<M\left|g^{\prime}\right|$, whence follows (1.6).

For the proof of Theorem 3 we note that, by the result [5, Theorem 5(ii), p. 627] due to G. H. Hardy and J. E. Littlewood, there exists $K \in \Lambda_{\alpha-1 / p}$ such that $k\left(e^{i t}\right)=K\left(e^{i t}\right)$ a.e. on $C$. Consequently, $K$ is bounded from above and from below,

$$
0<m \leqslant K\left(e^{i t}\right)<M<\infty \text { for all } e^{i t} \in C,
$$

where $m$ is the number in Theorem 3. Now, $T_{k}=T_{K}=M \exp G$, where

$$
G(z)=\frac{1}{2 \pi} \int_{-\pi}^{\pi} \frac{e^{i t}+z}{e^{i t}-z} \log \frac{K\left(e^{i t}\right)}{M} d t .
$$

Since $K \in \Lambda_{\alpha-1 / p}$, it follows from Theorem 2 that $T_{k}=T_{K}$ is continuous on $\bar{U}$ and that $\left|T_{k}^{*}\right|=\left|T_{K}^{*}\right|=K=k$ a.e. on $C$. For the proof of (1.7), we note that $\operatorname{Re} G$ is bounded because $|\log (K / M)| \leqslant \log (M / m)$. Therefore, $G$ is of Hardy class $H^{q}$ for all $q>0$ by [4, Theorem 4.1, p. 54]. Since $\log (k / M) \in \Lambda_{\alpha}^{p}$, it follows that $\log (K / M) \in \Lambda_{\alpha}^{p}$. Thus, by the $\Lambda_{\alpha}^{p}$ analogue of [4, Theorem 5.8, p. 83], being [4, Exercise 12, p. 91], one knows that $G^{*} \in \Lambda_{\alpha}^{p}$. Then by [4, Theorem 5.4, p. 78],

$$
\int_{-\pi}^{\pi}\left|G^{\prime}\left(r e^{i t}\right)\right|^{p} d t=O\left((1-r)^{-p+p \alpha}\right) .
$$

Again, by $\left|T_{k}^{\prime}\right|=\left|T_{K}^{\prime}\right| \leqslant M\left|G^{\prime}\right|$, together with (2.1), one observes that (1.7) holds.

\section{A concluding remark}

As far as the condition of the type (1.7) is concerned, the following might be noteworthy.

Suppose that $k \in \Lambda_{\alpha}^{p}(1<p<\infty, 0<\alpha<1)$ is real and that there exist constants $m>0$ and $M>0$ such that $m \leqslant k \leqslant M$ a.e. on $C$. Then

$$
\int_{-\pi}^{\pi}\left|T_{k}^{\prime}\left(r e^{i t}\right)\right|^{p} d t=O\left((1-r)^{-p+p \alpha}\right) \text { as } r \rightarrow 1-0 .
$$

For a sketch of the proof we set

$$
\Gamma(z)=\frac{1}{2 \pi} \int_{-\pi}^{\pi} \frac{e^{i t}+z}{e^{i t}-z} \log \frac{k\left(e^{i t}\right)}{M} d t .
$$


Then, $T_{k}=M \exp \Gamma$ and $\log (m / M) \leqslant \operatorname{Re} \Gamma \leqslant 0$. We can show that

$$
\int_{-\pi}^{\pi}\left|\Gamma^{\prime}\left(r e^{i t}\right)\right|^{p} d t=O\left((1-r)^{-p+p \alpha}\right),
$$

which, together with $\left|T_{k}^{\prime}\right| \leqslant M\left|\Gamma^{\prime}\right|$, proves (3.1).

\section{References}

[1] L. Carleson, 'A representation formula for the Dirichlet integral', Math. Z. 73 (1960), $190-196$.

[2] K.-N. Chow and D. Protas, "The bounded, analytic, Dirichlet finite functions and their fibers', Arch. Math. (Basel) 33 (1979), 575-582.

[3] J. A. Cima, 'A theorem on composition operators', Banach spaces of analytic functions, pp. 21-24 (Lecture Notes in Mathematics 604, Springer-Verlag (1977)).

[4] P. L. Duren, Theory of $H^{p}$ spaces (Academic Press, New York and London, 1970).

[5] G. H. Hardy and J. E. Littlewood, 'A convergence criterion for Fourier series', Math. Z. 28 (1928), 612-634.

Department of Mathematics

Tokyo Metropolitan University

Fukazawa, Setagaya

Tokyo 158, Japan 